\title{
Immediate Post-Operative Outcomesof Stapled and Hand Sewn Techniques during Oesophagogastrostomy: A Randomized Clinical Trial
}

\author{
Mohammad Zakir Hossain Bhuiyan', Syed Aminul Haque ${ }^{2}$, \\ Mohammad Ahtashamul Haque 3 , Farhana Tarannum ${ }^{4}$ \\ ${ }^{1}$ Assistant Professor, Department of Thoracic Surgery, Chittagong Medical College, Chittagong, Bangladesh; ${ }^{2}$ Associate \\ Professor, Department of Thoracic Surgery, Chittagong Medical College, , Chittagong, Bangladesh; ${ }^{3}$ Assistant \\ Professor, Department of Oral and Maxillofacial Surgery, Dhaka Medical College, Dhaka, Bangladesh; \\ ${ }^{4}$ Medical Officer, Raujan Health Complex, Raujan, Chittagong, Bangladesh
}

[Received: 12 August 2019; Accepted: 20 October 2019; Published: 1 January 2020]

\begin{abstract}
Background: Oesophagogastrostomy is a very crucial surgical management. Objective:The purpose of the present study was to observe the immediate post-operative outcomes of stapled and hand sewn techniques during oesophagogastrostomy. Methodology: This single centre, parallel randomized clinical trial was conducted in the Department of Thoracic Surgery at National institute of Diseases of the Chest and Hospital, Dhaka, Bangladesh from January 2011 to December 2011 for a period of one year. Prior to commencement of this study the study protocol was approved by the thesis committee of NIDCH. Patients were purposefully selected from different surgery ward of NIDCH irrespective of age and sex of patients for the study.Patients were divided into two groups designated as group A and B.Stapled oesophagogastric anastomosis was performed in group $\mathrm{A}$ and hand sewn anastomosis was done in group B.Immediate post-operative complication were recorded. Result: A total of 60 patients of oesophageal or gastric cardia carcinoma were recruited for this study. Of them 32 cases were assigned to Stapler method and 28 cases to Hand-sewn method of oesophagogastrostomy. The mean age of the patients of group A and group B were $56.1 \pm 12.7$ years and $55.3 \pm 8.3$ years respectively. Regarding blood loss during operation, the table shows that in group A mean + SD were $430.4 \pm 63.2 \mathrm{ml}$ and in group B mean + SD were $529.6+86.9 \mathrm{ml}$. In one stage operation in group A it was mean + SD i.e. $362.2 \pm 38.3 \mathrm{ml}$ and in group B it was mean + SD 529.6 $\pm 86.9 \mathrm{ml}$. In two stage operation in group A it was mean $466.2 \pm 39.7 \mathrm{ml}$ and in group B it was mean $\pm \mathrm{SD} 585.6 \pm 62.2 \mathrm{ml}$. Respiratory complication developed in $9.4 \%$ of the patients in group A and $17.9 \%$ patients in group B. Cardiac complication developed in $3.1 \%$ of the patients in group A and $7.1 \%$ of patients in group B. Wound infection developed in $12.5 \%$ of the patients in group A and $10.7 \%$ of the patients in group B. Conclusion: In conclusion most of the immediate anastomotic complications are less in stapled anastomotic technique than hand sewn technique. [Journal of National Institute of Neurosciences Bangladesh, 2020;6(1):43-47]
\end{abstract}

Keywords: Immediate; post-operative outcomes; stapled and hand sewn techniques; oesophagogastrostomy

Correspondence: Dr. Mohammad Zakir Hossain Bhuiyan,Assistant Professor, Department of Thoracic Surgery, Chittagong Medical College, Chittagong, Bangladesh; Email: drzakirdmc@yahoo.com; Cell no.: +8801712152051

Conflict of interest:There is no financial conflict of interest relevant to this paper to disclose.

Funding agency: This research project was not funded by any group or any institution.

Contribution to authors: Bhuiyan MZH,Haque SAcontributed from the protocol preparation, data collection up to report writing. Manuscript writing was performed by Bhuiyan MZH, Haque MA, Tarannum F have revised the manuscript.

How to cite this article: Bhuiyan MZH,Haque SA, Haque MA, Tarannum F. Immediate Post-Operative Outcomes of Stapled and Hand Sewn Techniques during Oesophagogastrostomy: A Randomized Clinical Trial. J NatlInstNeurosci Bangladesh, 2020;6(1):43-47 Copyright: (C2020. Bhuiyan et al. Published by Journal of National Institute of Neurosciences Bangladesh. This article is published under the Creative Commons CC BY-NC License (https://creativecommons.org/licenses/by-nc/4.0/). This license permits use, distribution and reproduction in any medium, provided the original work is properly cited, and is not used for commercial purposes.

\section{Introduction}

Surgical excision was previously considered as the main method for treating gastrointestinal (GI) tumors ${ }^{1}$. Anastomotic procedure is one of the key factors determining surgical success. Hand-sewn and stapled sutures comprise the major anastomotic methods in clinical practice of GI surgeries. esophagogastrostomy is performed for different surgical conditions of oesophagus ${ }^{2}$. There are different methods of oesophagogastrostomy. In transhiatal method, extirpation of the intrathoracico esophagus is done without a thoracotomy, and advancement of the oesophageal substitute, usually a greater curvature gastric tube is performed to the neck for reconstruction ${ }^{3}$.

The standard transthoracic oesophagogastrectomy is 
the most widely performed operation for cancer of the oesophagus worldwide ${ }^{4}$. This procedure can be carried out by a right or left thoracotomy depending on the preference of the surgeon and the localization of the tumour within the oesophagus. Generally a right thoracotomy is required for adequate exposure of tumours in the middle or upper third that are anatomically intimately related to the membranous trachea or the arch of the aorta. Tumours located at the gastrooesophageal junction or in the lower third of the oesophagus can usually be approached through a left thoracotomy incision combined with a left phrenotomy or, ahernatively, with a left thoracoabdominal incision ${ }^{5}$.

The surgical treatment of esophageal cancer remains controversial ${ }^{6}$. For example, various reconstructive options are available after esophagectomy for esophageal cancer. These options depend on the reconstructed organ (stomach, colon, or small intestine), characteristics of the esophageal conduit (whole stomach or thin gastric roll, jejunum or ileum, or left or right colon), location of anastomosis (cervical or thoracic), anastomotic method (hand-sewn or stapled), route of reconstruction (antethoracic, retrosternal, or postmediastinum). Of these factors, the anastomotic technique is obviously one of many variables that can affect the operative morbidity or postoperative course. In fact, hospital deaths after esophagectomy are related to postoperative impediments, such as pulmonary complications and anastomotic leakage ${ }^{7}$. Therefore, much effort has been devoted to reducing the occurrence of anastomotic leakage. This present study was undertaken to observe the immediate post-operative outcomes of stapled and hand sewn techniques during oesophagogastrostomy.

\section{Methodology}

Study Population and Settings: This single centre, parallel randomized clinical trial was conducted in the Department of Thoracic Surgery at National institute of Diseases of the Chest and Hospital, Dhaka, Bangladesh from January 2011 to December 2011 for a period of one year. Prior to commencement of this study the study protocol was approved by the thesis committee of NIDCH. All patients were informed about the study. Patients were purposefully selected from different surgery ward of NIDCH irrespective of age and sex of patients for the study.All the patients having carcinoma of oesophagus at the lower two third irrespective of age and sex undergoing surgery were included as study population. Patients having carcinoma in upper one third of oesophagus or having advanced stage (i.e. inoperable) of carcinoma of oesophagus were excluded from this study.

Randomization and Blinding: Patients were divided into two groups by computer generated simple random sampling technique into two groups designated as group A and B.

Allocation: Stapled oesophagogastric anastomosis was performed in group $\mathrm{A}$ and hand sewn anastomosis was done in group B.One-stage operation was carried out for the lesions in the lower third of oesophagus and at the oesophagogastric junction.Two-stage operation was carried out for the lesions in the middle third of oesophagus. The linear stapler was used to resect the diseased portion of the stomach and at the same time to anastomose the cut margins of it. The circular one was used for anastomosis between the oesophagus and the anterosuperior surface of the stomach.

Follow up and Outcome Measures: Approximate blood loss was also measured in both groups of patients in the two separate techniques and recorded. Techniques used for measuring blood loss were traditional methods. The methods were weighing swabs (mops and gauges)before and after soaking by blood. Lost blood in the operative field was sucked into a measuring jar and amount of blood was measured in this way.

Statistical analysis: Collected data were expressed as mean + SD and mean \pm SE. The effect of variables were analyzed using standard statistical method using SPSS. Data were recorded including patient's demographic details, hospital resource utilization, length of stay and other measures of variables. Student pair ( $\mathrm{t}$ ) test was used. A value of ' $\mathrm{p}$ ' is equal to or $<0.05$ was considered to be significant.

\section{Results}

A total of 60 patients of oesophageal or gastric cardia carcinoma were recruited for this study. Of them 32 cases were assigned to Stapler method and 28 cases to Hand-sewn method of oesophagogastrostomy.The mean age of the patients of group A and group B were $56.1+12.7$ years and $55.3+8.3$ years respectively (Table 1). 
Table 1: Demographic Variables of Patients

\begin{tabular}{lcc}
\hline Group & Age (Mean \pm SD) & P value \\
\hline Group A & $56.1 \pm 12.7$ & \\
Group B & $55.3 \pm 8.3$ & 0.787 \\
\hline
\end{tabular}

*Student's t-Test was done to see the level of significance

In group $\mathrm{A}$, one stage operation was performed in $34.4 \%$ and two stage operation was performed in $65.6 \%$ cases. In group B, it was $42.9 \%$ and $57.1 \%$ for one stage and two stage operation respectively (Table 2).

Table 2: Distribution of patients by type of operation performed

\begin{tabular}{lccc}
\hline \multirow{2}{*}{$\begin{array}{l}\text { Type of } \\
\text { Operation }\end{array}$} & \multicolumn{2}{c}{ Group } & P value \\
\cline { 2 - 3 } & Group A & Group B & \\
\hline One stage & $11(34.4)$ & $12(42.9)$ & \\
Two stage & $21(65.6)$ & $16(57.1)$ & 0.500 \\
Total & $\mathbf{3 2}(\mathbf{1 0 0 . 0 \% )}$ & $\mathbf{2 8 ( 1 0 0 . 0 \% )}$ & \\
\hline
\end{tabular}

Regarding blood loss during operation, the table shows that in group A mean + SD were $430.4+63.2 \mathrm{ml}$ and in group B mean $+\mathrm{SD}$ were $529.6+86.9 \mathrm{ml}$. In one stage operation in group A it was mean + SD i.e. $362.2+$ $38.3 \mathrm{ml}$ and in group B it was mean + SD $529.6+86.9$ $\mathrm{ml}$. In two stage operation in group $\mathrm{A}$ it was mean $466.2+39.7 \mathrm{ml}$ and in group B it was mean $+\mathrm{SD}$ $585.6+62.2 \mathrm{ml}$. Duration of operation shows that in group A mean \pm SD were $204.6+39.3$ min. In group B it was mean + SD $251.7+36.3$ min.In group A (one stage) mean $+\mathrm{SD}$ were $167.2+9.9$ min while in group $\mathrm{B}$ it was mean $+\mathrm{SD}$ were $212.5+8.6$ min. In group A(two stage) mean \pm SD $224.3+26.1$ min while in group B it was $281.2+12.7$ min. (Table 3 ).

Table 3: Distribution of patients by peroperative findings

\begin{tabular}{lccc}
\hline Peroperative & \multicolumn{2}{c}{ Group } & Pvalue \\
\cline { 2 - 3 } findings & Group A & Group B & \\
\hline Blood loss* & $430.4 \pm 63.2$ & $529.6 \pm 86.9$ & $<0.001$ \\
One stage* (ml) & $362.2 \pm 38.3$ & $454.9 \pm 51.4$ & $<0.001$ \\
Two-stage* (ml) & $466.2 \pm 39.7$ & $585.6 \pm 62.2$ & $<0.001$ \\
Duration of operation* & $204.6 \pm 39.3$ & $251.7 \pm 36.3$ & $<0.001$ \\
One stage* (min) & $167.2 \pm 9.9$ & $212.5 \pm 8.6$ & $<0.001$ \\
Two-stage* (min) & $224.3 \pm 26.1$ & $281.2 \pm 12.7$ & 0.001 \\
\hline
\end{tabular}

* Student's t-Test was done to analyse the data and data were presented as mean $\pm \mathrm{SD}$.

Respiratory complication developed in $9.4 \%$ of the patients in group A and $17.9 \%$ patients in group B. Cardiac complication developed in $3.1 \%$ of the patients in group A and $7.1 \%$ of patients in group B.
Wound infection developed in $12.5 \%$ of the patints in group $\mathrm{A}$ and $10.7 \%$ of the patients in group B. Anastomotic leakage developed in $3.1 \%$ and $10.7 \%$ of the patients in group A and group B respectively. Reoperation for bleeding was done in one .(3.6\%) patients in group B. The average hospital stay was 13.4 days in group A and 13.7 days in group B $(\mathrm{p}=0.696)$. Two patients in each group died during their hospital stay (Table 4).

Table 4: Distribution of patients by in-hospital complications between groups

\begin{tabular}{lccc}
\hline \multirow{2}{*}{$\begin{array}{l}\text { Variables of } \\
\text { morbidity }\end{array}$} & \multicolumn{2}{c}{ Group } & P value \\
\cline { 2 - 3 } & Group A & Group B & \\
Respiratory complication* & & & \\
Yes & $3(9.4)$ & $5(17.9)$ & 0.280 \\
No & $29(90.6)$ & $23(82.1)$ & \\
Cardiac complication* & & & \\
Yes & $1(3.1)$ & $2(7.1)$ & 0.449 \\
No & $31(96.9)$ & $26(92.9)$ & \\
Wound infection* & & & \\
Present & $4(12.5)$ & $3(10.7)$ & 0.577 \\
Absent & $28(87.5)$ & $25(89.3)$ & \\
Anastomotic leakage* & & & \\
Yes & $1(3.1)$ & $3(10.7)$ & 0.257 \\
No & $31(96.9)$ & $25(89.3)$ & \\
Re-operation for bleeding* & & & \\
Yes & $0(0.0)$ & $1(3.6)$ & 0.467 \\
No & $32(100) .0)$ & $27(96.4)$ & \\
Hospital stay\# (days) & $13.4 \pm 3.5$ & $13.7 \pm 2.2$ & 0.696 \\
Mortality before discharge* & & & \\
Yes & $2(6.3)$ & $2(7.1)$ & 0.641 \\
No & $30(93.7)$ & $26(92.9)$ & \\
\hline
\end{tabular}

*Data were analysed using Fisher's Exact Test.

\#Student's t-Test was done to analyse the data and data were presented as mean $\pm \mathrm{SD}$.

\section{Discussion}

Oesophageal cancers are highly lethal neoplasm having a poor prognosis 8 . Despite advances in multimodality therapy, 5 year survival generally remains less than $10 \%$ cases $^{9}$. But in spite of the operative risks, surgery remains the primary mode of therapy for carcinoma of the oesophagus \& oesophagogastric junction because of the poor cure rate and persistence of symptoms after therapy with other modalities ${ }^{10}$.

In Bangladesh there are some studies regarding oesophageal carcinoma which have been done previously but unfortunately there is no study regarding comparison of outcome of oesophagogastrostomy after oesophagogastrectomy between stapler and hand sewn 
technique ${ }^{11}$. Previous studies in Bangladesh showed that the incidence of carcinoma oesophagus to be $5.8 \%$ among males and $4.24 \%$ among females in all cancer patients who attended in the radiology department of Dhaka Medical College Hospital ${ }^{12}$. Later, $\mathrm{Kabir}^{13}$ carried out a study in NIDCH where it has been reported that carcinoma oesophagus may hold $6^{\text {th }}$ or, $7^{\text {th }}$ position in the order of frequency when all the cancer patients in Bangladesh are considered.

In the present study, 60 patients of the oesophageal carcinoma were assessed. This study was conducted in the National Institute of Diseases of the Chest and Hospital, from January 2011 to December 2011. Patients having resectable malignant lesion in the middle and lower third of the oesophagus and oesophagogastric junction who underwent oesophagogastrectomy and oesophagogastrostomy were included in this study. This study was particularly designed to analyze the short term outcome of oesophagogastrectomy with oesophagogastrostomy for carcinoma of the oesophagus and cardia and to compare the results between the stapling and hand sewn technique of anastomosis.

In this study, the mean age of the subjects was $56.1 \pm 12.7$ years in group A and $55.3 \pm 8.3$ years in group B. The lowest and highest ages were 25 years and 71 years respectively. A total of 41 patients $(68.3 \%)$ were in between 51 to 60 years. Bruni and Nelson ${ }^{14}$ studied a total of 113 cases with a diagnosis of carcinoma of the esophagus and cardia and it showed that most of them were between 50 to 70 years of age. More recently Karl et a ${ }^{15}$ collected data of 143 patients who underwent oesophageal resection for cancer of the esophagus and oesophagogastric junction and demonstrated that the age at the time of surgery averaged 63.7 years, ranging from 33 years to 83 years. All of these were consistent with the age distribution of the current series.

In this study, 37(61.67\%) patients underwent two stage oesophagogastrectomy with oesophagogastrostomy (both group A and group B) while the rest of the patients, 23(28.33\%) cases experienced one stage operation (both groups). Type of operation performed was depended on the site of lesion. One stage operation was done for the lesion in the lower third and cardia while two stage operations was for the lesions in the middle third. Kabir ${ }^{13}$ in his study showed that the number of one stage operation done was 23(48.93 \%) and number of two stage operation done was 24 $(51.10 \%)$ which is almost equal. However, on the contrary in our study two stage operations was done more frequently. Malcolm et $\mathrm{al}^{4}$ reported in their study that $61 \%$ underwent a left oesophagogastrectomy (one stage operation) and $37 \%$ cases two-stage operation. However, staging is not mandatory to be compared as most of the patients had middle third lesion and so we had to choose the two stage operations it is dependent on the site of lesion.

Blood loss during operation in one stage operation was $362.2 \pm 38.3 \mathrm{ml}$ in group A while $454.9 \pm 51.4 \mathrm{ml}$ in group B. In two stage population, $466.2 \pm 39.7 \mathrm{ml}$ in group A and $585.6 \pm 62.2 \mathrm{ml}$ in group B. In another word, average blood loss in group A is $430.6 \pm 63.2 \mathrm{ml}$ in comparison with $529.6 \pm 86.9 \mathrm{ml}$ in group B. Shanda et $\mathrm{al}^{18}$ (2007) showed that an estimated blood loss in group A was $435 \pm 240 \mathrm{ml}$ while $640 \pm 350 \mathrm{ml}$ in group B. The blood loss in our study is almost equal in stapling group but little less in hand sewn group than that of the above mentioned study.

This present study demonstrates that among the post-operative complication, respiratory complication was the commonest one; i.e. in 8 patients $(13.33 \%$, $\mathrm{n}=60)$. Of these 3 patients $(9.4 \%, \mathrm{n} 1=32)$ belong to group A and 5 patients $(17.9 \%, \mathrm{n} 2=28)$ belong to group B. Respiratory complications include respiratory failure, pleural effusion, pneumothorax, atelectasis and pneumonitis. Out of these 8 patients, 3 patients needed ventilator support, 2 of them died (one from each group). The next common complication was found wound infection, 7 patients $(11.67 \%, \mathrm{n}=60)$ had suffered from this complication. Of these, 4 patients $(12.5 \%, \mathrm{n} 1=32)$ belong to group $\mathrm{A}$ and 3 patients $(10.7 \%, \mathrm{n} 2=28)$ belong to group $\mathrm{B}$. The most grave complication was anastomotic leakage, 1 patient $(3.1 \%$, $\mathrm{n} 1=32)$ in group $\mathrm{A}$ and 3 patients $(10.7 \%, \mathrm{n} 2=28)$ in group B. 2 patients died (one from each group) from this complication due to intractable mediastinitis. I patient $(3.1 \%, \mathrm{n} 1=32)$ from group $\mathrm{A}$ and 2 patients $(7.1 \%, \mathrm{n} 2=28)$ from group $\mathrm{B}$ developed cardiac complication, who were treated conservatively but no casualty happened from this complication. Re operation had to be done in I case $(3.1 \%, \mathrm{n} 2=28)$ for bleeding who belongs to group B. Hospital stay for group A was $13.4 \pm 3.5$ days whereas $13.7 \pm 2.2$ days for group B. There was no gross difference between the groups regarding hospital stay.

Mortality before discharge was 4 patients $(6.7 \%, n=60)$ due to respiratory complication and anastomotic leakage which have already been discussed earlier. Two patients in each group died of these complications. In the study by $\mathrm{Kabir}^{13}, 13(27.7 \%)$ patients had respiratory complications, $5(10.6 \%)$ had anastomotic 
leakage and $5(10.6 \%)$ had gross electrolyte imbalance. Total number in-hospital death was $11(31.4 \%)$. Our data of respiratory complications and anastomotic leakage are almost similar to him but we had a lesser death rate. Zhang et $\mathrm{al}^{11}$ showed that anastomotic leakage in group A was $6(2.2 \%)$ and I (0.4\%) in group B. They also showed that 30 day post-operative mortality was $7(2.6 \%)$ in group A and $3(1.2 \%)$ in the group B. This finding is mildly less than that of our findings i.e. $6.25 \%$ in group A and $7.14 \%$ in group B. But in the previous study, death rate was much more than that of our study. The overall lower rates of anastomotic leaks, respiratory complications, cardiac complications are found in the group A than that of group B. But wound infection was slightly more in group A than that group B.

The rate of anastomotic leakage of the anastomosis between the remnant cervical esophagus and esophageal substitute is higher than that of other type of gastrointestinal anastomosis. To date, many studies have compared the anastomotic complication of the hand-sewn or stapled anastomosis. Zhu et $\mathrm{al}^{16}$ reviewed the major outcomes of the nonrandomized or randomized control trials of esophagogastric anastomosis after esophagectomy for esophageal cancer. In this review, several reports of nonrandomized studies described a decreased rate of anastomotic leakage with stapled anastomosis compared to hand-sewn anastomosis. However, none of the randomized control trials reported statistically significant differences in the rate of anastomotic leakage, which varied depending on the reconstructed organs, approach, or anastomotic technique.

\section{Conclusion}

In conclusion most of the immediate anastomotic complications are less in stapled anastomotic technique than hand sewn technique. Furthermore the cardiorespiratory complications are also less in number in stapled technique. Even wound infection is also few in number in stapled technique. Large scale study should be performed to see the real scenario.

\section{References}

1. Islam KS 2008, 'Oesophagogastrectomy with oesophagogastrostomy for carcinoma of the oesophagus and oesophagogastric junction- early result', MS thesis, Bangabandhu Sheikh Mujib Medical University, Dhaka, Bangladesh

2. Law S, Fok M, Chu KM, Wong J, 1997, 'Comparison of hand-sewn and stapled esophagogastric anastomosis after esophageal resection for cancer; a prospective randomized controlled trial' Ann Surg, 1997 Aug; 226 (2); 169-73.

3. Luechakiettisak P and Suppapong K 2008, 'Comparison of hand sewn and stapled in esophagogastric anastomosis after esophageal cancer resection: A prospective Randomized study' Journal of the Medical association of Thailand, vol 91, no 5, pp $681-685$.

4. Malcolm JR, Dalrymple-Hay, Evans KB and Richard EL, 1999, 'Oesophagectomy for carcinoma of the oesophagus and oesophagogastric junction', European Journal of Cardio-Thoracic Surgery, vol.15, pp. 626-630.

5. Nasser KA 2008, ' Primary surgery for adenocarcinoma of the esophagus', in: G. Alexander Patterson, Joel D. Cooper, Jean Deslauriers, Antoon (Toni) EMR, Lerut James D, Luketich Thomas W, Rice, eds. Pearson's thoracic and esophageal surgery, 3`d edition, Churchill, Livingstone, Philadelphia, pp 486 - 490.

6. Sarma SK 1992, 'Distribution pattern of 3399 new cancer patient- a one year study', Bangladesh Medical Journal, Khulna Branch, vol.25, pp. 16-18.

7. Shanda HB, Arlene M, Correa, Bobb W, Wayne LH, Linda WM, et al, 2007, 'Propensity matched analysis of three techniques for' intra thoracic esophagogastri anastomosis', Ann Thoracic Surg, vol 83, pp $1805-1813$.

8. Sina E, Thomas WR, Sudish CM, Lisa AR, Eugene HB, 2005, Does esophagogastric anastomotic technique influence the outcome of patients with esophageal cancer?', J Thoracic CardiovascSurg, vol 129, pp 623 - 631.

9. Swisher, Hunt, Holmes, Zinner and McFadden, 1995, 'Changes in the Surgical Management of Esophageal Cancer from 1970 to 1993', Am. J. Surg., vol.169, pp. 649-614.

10. Urschel JD, 1995, Esophagogastrostomy anastomotic leaks complicating esophagectomy: a review. Am J Surg; vol. 169: 634 640

11. Zhang YS, Gao BR, Wang HJ, 2010, Layered versus stapler oesophagogastric anastomosis, The Journal of International Medical Research, 38: 227 - 233.

12. Sharpe DAC and Moghissi K, 1996, 'Resectional surgery in carcinoma of the oesophagus and cardia: what influences long-term survival?' Eur J Cardio-thoracSurg, vol.10, pp. 359-364. 13. Kabir J, 1994, 'Esophageal carcinoma: clinical profile, surgical management and follow $\neg$ up', MS thesis, the university of Dhaka, Bangladesh.

14. Bruni HC and Nelson RS, 1975, 'Carcinoma of the esophagus and cardia: diagnostic evaluation in 113 cases', The Journal of Thoracic and Cardiovascular Surgery, vol.70, pp.367-369

15. Karl, Schreiber, Boulware, Baker and Coppola, 2000, 'Factors Affecting Morbidity, Mortality and Survival in Patients Undergoing Ivor Lewis Esophagogastrectomy', Ann Surg, vol.231, pp. 635-643

16. Zhu ZJ, Zhao YF, Chen LQ, 2008, Clinical application of layered anastomosis during oesophagogastrectomy. World Journal Surg; 32: $583-588$ 